\title{
Reduced graphene oxide obtained using the spray pyrolysis technique for gas sensing
}

\author{
O.M. Slobodian ${ }^{1 *}$, Yu.S. Milovanov ${ }^{2}$, V.A. Skryshevsky ${ }^{2}$, A.V. Vasin ${ }^{1}$, X. Tang ${ }^{3}$, J.-P. Raskin ${ }^{3}$, P.M. Lytvyn ${ }^{1}$, \\ K.V. Svezhentsova ${ }^{1}$, S.V. Malyuta ${ }^{1}$, A.N. Nazarov ${ }^{1}$ \\ ${ }^{I}$ V. Lashkaryov Institute of Semiconductor Physics NAS of Ukraine, Kyiv, Ukraine \\ ${ }^{2}$ Institute of High Technologies Taras Shevchenko National University of Kyiv, Kyiv, Ukraine \\ ${ }^{3}$ ICTEAM, Université catholique de Louvain (UCLouvain) Louvain-la-Neuve, Belgium \\ *Corresponding author e-mail: akapulko20@gmail.com
}

\begin{abstract}
Graphene oxide films were formed using the ultrasonic spray coating method and studied with micro-Raman spectroscopy, atomic force microscopy, and electrical dynamic response of resistance measurements. Effect of different gases (water vapor, ethanol, acetone, ammonia, and isopropyl) on the dynamic response of resistance of the $\mathrm{Au}$ / graphene oxide / Au structure has been studied. The dynamic response shows that adsorption of all mentioned gases results in increase of the resistance. For ethanol, acetone and isopropyl adsorption and desorption cycles are almost identical. At the same time, in the case of water vapor and ammonia the cycle of desorption is very week, especially for the former, which attests different mechanisms of adsorption/desorption processes regarding to ethanol, acetone and isopropyl. The mechanisms of studied vapors adsorption/desorption are proposed.
\end{abstract}

Keywords: ultrasonic spray pyrolysis, graphene oxide, Raman spectroscopy, gas sensor.

doi: https://doi.org/10.15407/spqeo22.01.98

PACS 07.07.Df, 77.84.Bw, 78.30.Ly, 82.30.Lp

Manuscript received 14.02.19; revised version received 06.03.19; accepted for publication 27.03.19; published online 30.03.19.

\section{Introduction}

In recent years, the interest in nanostructured gas-sensing materials, particularly in thin film form, has significantly grown up. The devices based on them have good response, wide operation temperature range and stable selectivity. Moreover, sensors based on thin film materials are essential for mass production technologies. For industrial applications, a low cost method, specifically an ultrasonic spray pyrolysis technique, appears to be an excellent choice. The ultrasonic spray pyrolysis method is based on formation of fine droplets with size distribution in the range of a few micrometers. Hence, the ultrasonic spray pyrolysis method could be the best approach for deposition of uniform thin films.

At the same time, graphene and graphene-based materials are considered as promising candidates for advanced applications in future electronics [1-3] because of their attractive physical and optical properties. It was found that graphene oxide (GO) shows good sensing properties towards $\mathrm{H}_{2} \mathrm{O}$ [4, 5], $\mathrm{NO}_{2}$ [6], $\mathrm{NH}_{3}$ [7], $\mathrm{H}_{2}$ [8] and other gases and vapors.
However, graphene oxide changes its physical properties strongly in dependence on type of reduction. Well reduced graphene oxide (with a small number of $\mathrm{C}-\mathrm{O}$ and $\mathrm{C}-\mathrm{OH}$ bonds) can be obtained at hightemperature vacuum annealing [9] or low-temperature chemical reduction [10]. Additionally, it was shown that low-temperature annealing in air can result in appreciable reduction of resistivity of the GO [11], which is necessary for resistance sensing material. This method for synthesizing reduced graphene oxide ( $\mathrm{rGO})$ is the most simple and convenient one, but it is necessary to check up its performance for obtaining the sensory material.

In this work, we report GO-based sensors prepared by ultrasonic spray pyrolysis technique. The nanostructured GO is characterized by Raman spectroscopy and atomic force microscopy. The sensor resistances are measured by current-voltage characteristic in direct current mode, and measurements of current at vapor/dry air cyclic pumping (dynamic response) are performed. The sensors are used to detect ethanol, acetone, ammonia, isopropyl and water vapor. 


\section{Experimental}

GO was synthesized using Hummers' method [12] and transformed into water solution. Evolution of chemical composition of this material, after annealing, was determined using XPS and FTIR spectroscopy, as reported in [13]. The carbon/oxygen ratio of pristine GO was found to be 2.31 , which is in agreement with values reported for similar oxidation processes $[14,15]$. Then, an aqueous solution of GO was deposited onto the substrate. As shown in Fig. 1, the substrate is an Au interdigitated electrode array (IDA) on $\mathrm{SiO}_{2} / \mathrm{Si}$. Deposition (see Fig. 2) was performed using the ultrasonic spray coating technique with $120 \mathrm{kHz}$ atomizer nozzle (Sono-Tek Corp., USA), power level $-6 \mathrm{~W}$, flow rate $-3 \mathrm{ml} / \mathrm{h}$, for $5 \mathrm{~min}$. The substrate was located on a heated table (80$100{ }^{\circ} \mathrm{C}$ ). To focalize the beam of drops, the focusing air shroud was made and used. In this way, GO flakes are immobilized on IDA after solvent evaporation [16]. To obtain reduced GO film (rGO), the samples were annealed at $230{ }^{\circ} \mathrm{C}$ for $15 \mathrm{~min}$ in ambient air.

Both measurements of current-voltage characteristic in direct current mode and measurements of current at vapor/dry air cyclic pumping (dynamic response) were performed using Agilent 4156C semiconductor parameter analyzer. The nano-relief and homogeneity of GO film surfaces were analyzed using the FemtoScan atomic force microscope (AFM) operating in the contact mode (Fig. 1). The control of GO layer quality was fulfilled by micro-Raman spectroscopy at room temperature (triple Raman spectrometer T-64000 Horiba Jobin-Yvon, equipped with electrically cooled CCD detector, and excited by the 515-nm line of an Ar-Kr ion laser).

\section{Results and discussion}

\subsection{Structure and surface morphology of GO film}

AFM surface topology of GO film onto glass substrate, obtained with ultrasonic pyrolysis method, is presented in Fig. 3. GO films were aggregated to form clusters with the lateral sizes ranged from $500 \mathrm{~nm}$ up to several micrometers and the minimum thickness of about $3 \mathrm{~nm}$.

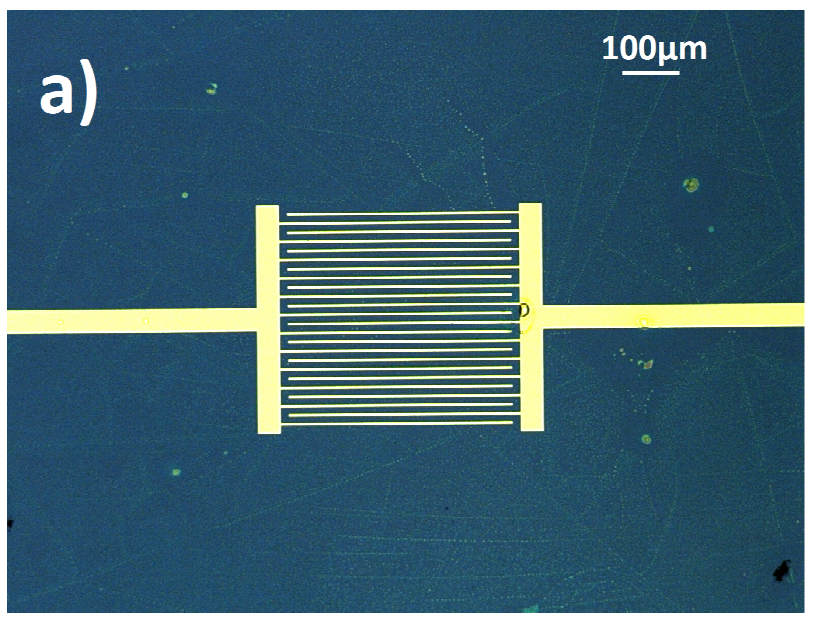

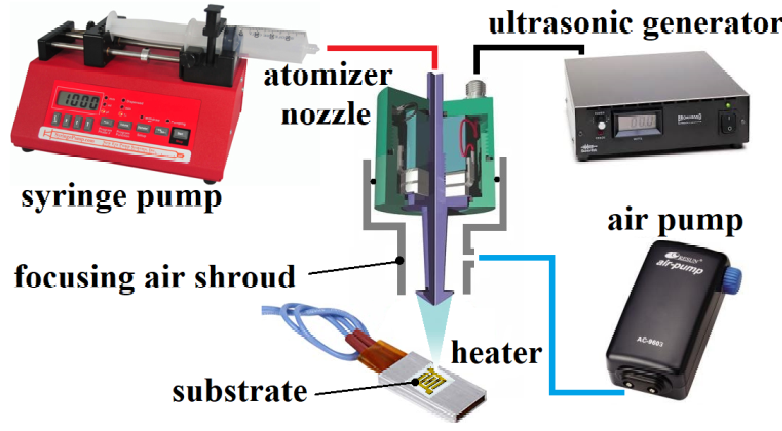

Fig. 2. Structural scheme of the ultrasonic pyrolysis system.

The root mean square (RMS) value of the GO height, averaged over an operation area $8 \times 8 \mu^{2}$, equals to $5.4 \mathrm{~nm}$. However, the local height of sharp thresholds between plateaus and valleys can reach 10-15 nm. The friction force map (Fig. 3b) illustrates a general homogeneity of mechanical and phase properties over the surface. At the same time, there is a local friction contrast of opposite sign (dark/light). The dark contrast associated with hole-like depressions in the relief (diameter $100 \ldots 300 \mathrm{~nm}$ ), where tip apex sticking and light contrast revealed fibrillar features of surface (wire-like features of $10 \times 100 \mathrm{~nm}$ and length $1-2 \mu \mathrm{m}$ ) with a small friction. Probably, these fibrillar features are the multiple wrinkles formed at GO flakes spraying.

Raman spectra of GO and rGO films are presented in Fig. 4. Two typical peaks, with their maxima at $\sim 1350$ and $\sim 1590 \mathrm{~cm}^{-1}$ (D and $\mathrm{G}$ bands, correspondingly) are observed. Weak changes of $I_{\mathrm{D}} / I_{\mathrm{G}}$ ratio from 0.92 to 0.91 could be associated with defect concentration growth after thermal treatment $[13,17]$, at the same time an oxidation degree remains significant [13].

\subsection{Dynamic response of $\mathrm{GO}$ film resistance}

The results of Raman measurements are confirmed by the relatively high resistance of the samples (see Fig. 5). Additionally, the current-voltage $(I-V)$ characteristics of the samples are non-linear for investigated range of

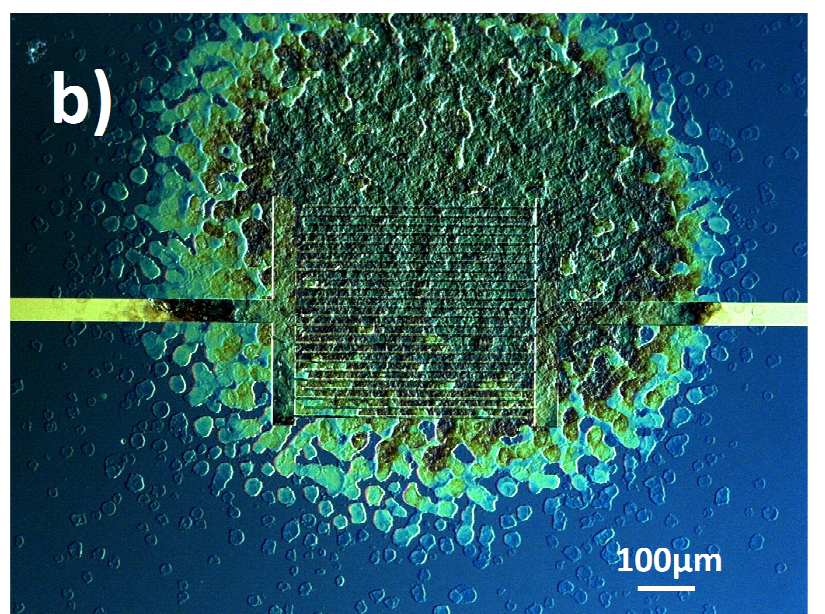

Fig. 1. Microscopic photo of Au electrodes (a) before, and (b) after spray deposition of GO film. 

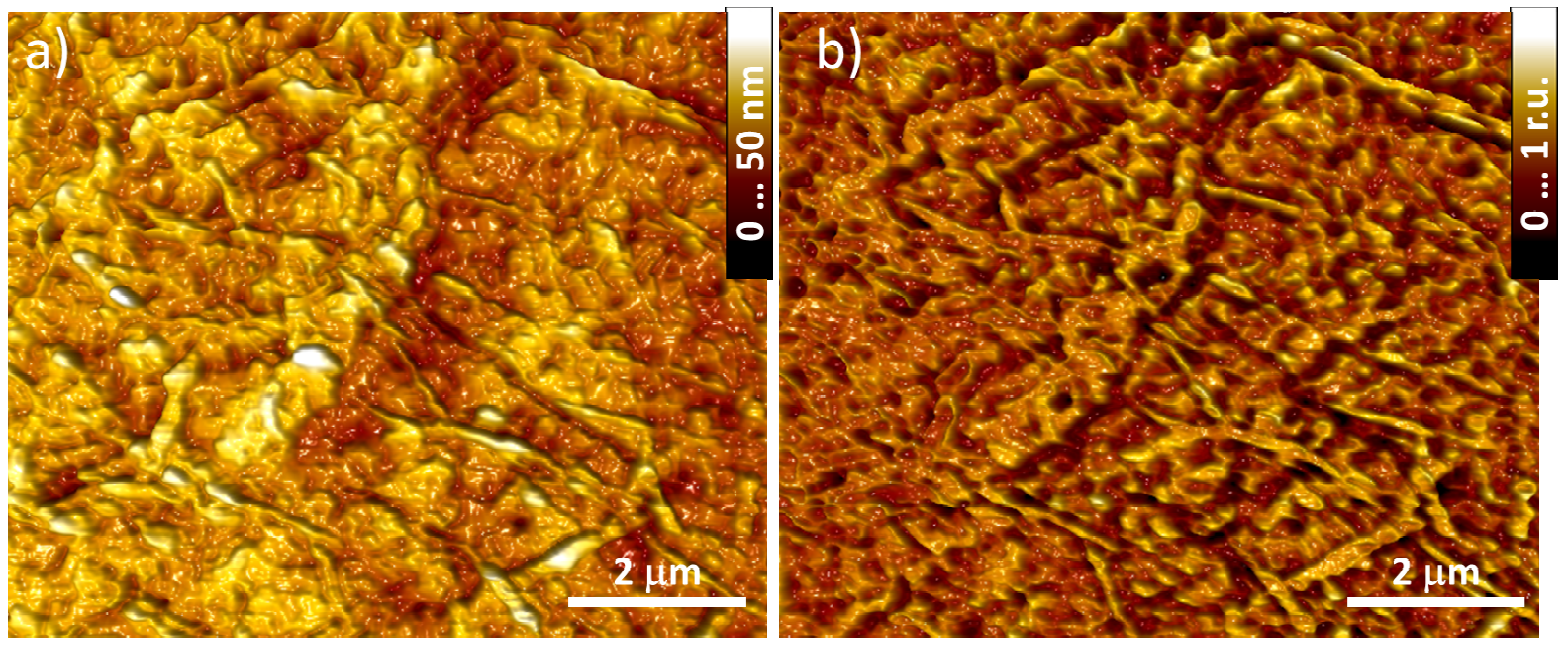

Fig. 3. AFM height (a) and friction (b) maps of the GO thin film deposited onto glass substrate with ultrasonic pyrolysis technique.

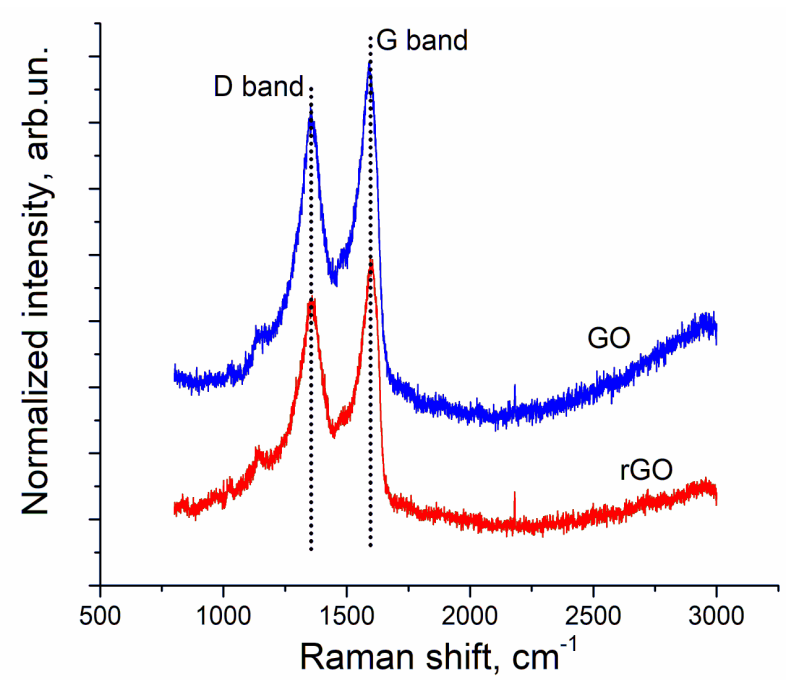

Fig. 4. Raman spectra of graphene oxide film before and after reduction.

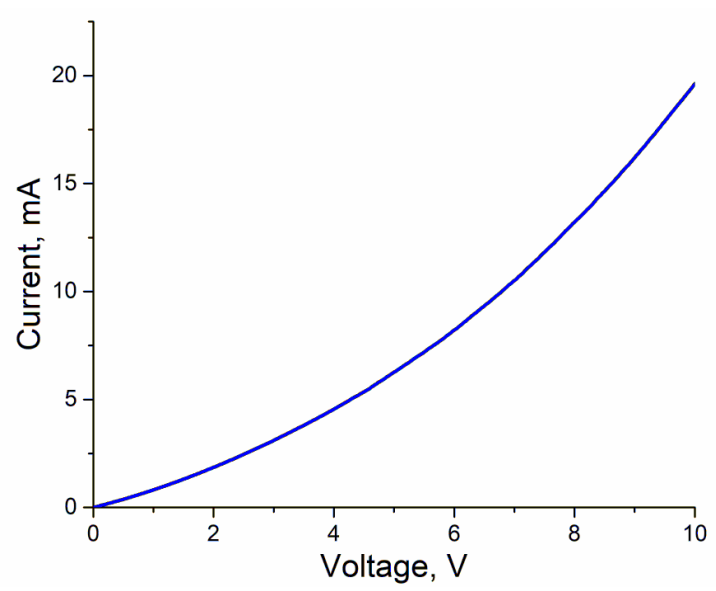

Fig. 5. $I-V$ characteristic of a reduced graphene oxide film device (see Fig. 1). applied voltage that can be associated with Schottky contact formation between $\mathrm{Au}$ and rGO.

Dynamic responses of the rGO film for ethanol, acetone, isopropyl, ammonia and water vapor are presented in Fig. 6. It can be seen all vapors under study (Fig. 6a-6e) provide the resistance increase in the rGO film. Additionally, the rGO film demonstrates the resistance responses $\left(\Delta R / R_{0}\right)$, to isopropyl of about $4 \ldots 5 \%$ (Fig. 6a), to ethanol about $5 \%$ (Fig. 6e), to ammonia about $15 \ldots 18 \%$ (Fig. 6c) and to water about $25 \ldots 30 \%$ in the first adsorption cycle (Fig. 6d). However, $\Delta R / R_{0}$ to acetone vapor is much lower, about $1 \ldots .2 \%$ (Fig. $6 \mathrm{~b}$ ).

It should be noted that at room temperature adsorption of most molecules (ethanol, isopropyl, acetone, ammonia) do not reach saturation after $800 \mathrm{~s}$, and desorption starts to appear. However, water vapor is in saturation at $200 \mathrm{~s}$, and week desorption is observed (Fig. 6d). These facts suggest the adsorption/desorption difference between water and the molecules mentioned above. Probably, incorporation of water molecules into the interlayer regions of the multilayer $\mathrm{rGO}$ reduces electrical contacts between rGO flakes, hereby, increasing the resistance of the rGO film [18]. Desorption of interlayer water in GO film is observed at temperatures about $100{ }^{\circ} \mathrm{C}[13]$.

The sensing mechanism for organic vapors, namely: ethanol $\left(\mathrm{C}_{2} \mathrm{H}_{5} \mathrm{OH}\right)$, acetone $\left(\left(\mathrm{CH}_{3}\right)_{2} \mathrm{CO}\right)$ and isopropyl $\left(\mathrm{C}_{3} \mathrm{H}_{7} \mathrm{OH}\right)$, is a complex process. Adsorption of organic vapors on rGO surface occurs through dissociation of the organic molecules to $\mathrm{H}^{+}$or $\mathrm{OH}^{-}$ions to form many different intermediate states. The final reactions of ethanol and acetone with adsorbed oxygen species can be described in the following form [19]:

$\mathrm{C}_{2} \mathrm{H}_{5} \mathrm{OH}(a d s)+6 \mathrm{O}^{-}(a d s) \rightarrow 2 \mathrm{CO}_{2}+3 \mathrm{H}_{2} \mathrm{O}+6 e^{-}$,

$\left(\mathrm{CH}_{3}\right)_{2} \mathrm{CO}(a d s)+8 \mathrm{O}^{-}(a d s) \rightarrow 3 \mathrm{CO}_{2}(g)+3 \mathrm{H}_{2} \mathrm{O}(g)+8 e^{-}$.

Slobodian O.M., Milovanov Yu.S., Skryshevsky V.A. et al. Reduced graphene oxide obtained using the spray ... 


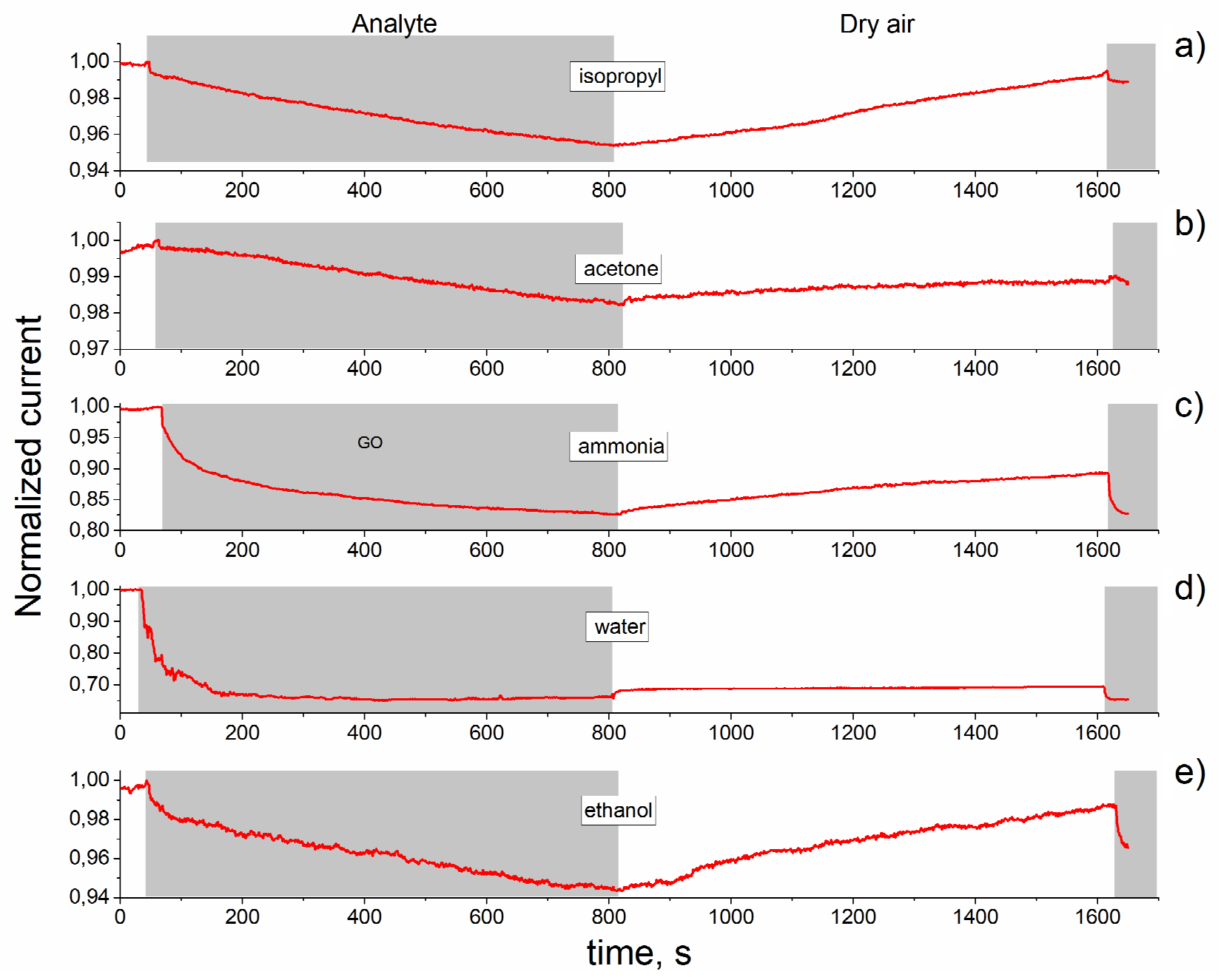

Fig. 6. Time dependence of normalized current (for $1 \mathrm{~V}$ applied voltage) for rGO samples, measured under (a) isopropyl, (b) acetone, (c) ammonia (d) water and (e) ethanol vapor.

Using the approach described in [19], the final reaction of isopropyl with adsorbed oxygen species can be also described by the following reaction:

$$
\mathrm{C}_{3} \mathrm{H}_{7} \mathrm{OH}(a d s)+9 \mathrm{O}^{-}(a d s) \rightarrow 3 \mathrm{CO}_{2}(g)+4 \mathrm{H}_{2} \mathrm{O}(g)+9 e^{-} .
$$

The similar approach can be used to explain ammonia reaction on surface of $\mathrm{rGO}$ that contains adsorbed oxygen [20]:

$$
\mathrm{NH}_{3}(a d s)+3 \mathrm{O}^{-}(a d s) \rightarrow \mathrm{N}_{2}(g)+3 \mathrm{H}_{2} \mathrm{O}(g)+3 e^{-} .
$$

The sensing mechanism of ethanol, acetone, isopropyl and ammonia is based on the fact that electrons from the chemical reactions mentioned above transfer to the graphene, which results in hole depletion of $p$-type graphene, thereby, increasing the resistance of the graphene film. If we compare the chemical reactions (1)(4), we can conclude that for the reaction (4) we need only three charged oxygen on the graphene surface whereas for ethanol - six ones. That is, the reaction (4) can performs faster than reactions (1)-(3). Indeed, resistance sensitivity of $\mathrm{rGO}$ film to ammonia is considerably higher than to ethanol and acetone.

\section{Conclusions}

The rGO films were formed using the ultrasonic spray coating method with low-temperature $\left(230^{\circ} \mathrm{C}\right)$ annealing in ambient atmosphere. It was shown that adsorption of such gases as ethanol, acetone, isopropyl, ammonia and water vapor results in increase of the resistance inherent to the rGO film. The water vapors demonstrate the highest sensitivity on the first step of adsorption, but a small desorption effect is observed. It is suggested that incorporation of water molecules into the interlayer regions of the multilayer rGO reduces electrical contacts between $\mathrm{rGO}$ flakes and increases of resistance of the rGO film. At room temperature, the maximum sensitivity for other studied gases is observed for ammonia which reaches $18 \%$. 


\section{Acknowledgements}

The authors thank Dr. A. Nikolenko from laboratory of Raman and Luminescence Submicron Spectroscopy (ISP NASU) for performance of the mirco-Raman measurements. Authors acknowledge the Ministry of Education and Science of Ukraine (Project F2211) and Ukrainian Scientific and Technology Centre (Project №6362) for partial support.

\section{References}

1. Avouris P., Xia F. Graphene applications in electronics and photonics. MRS Bullet. 2012. 37. P. 12251234. https://doi.org/10.1557/mrs.2012.206.

2. Choi W., Lahiri I., Seelaboyina R., Kang Y.S. Synthesis of graphene and its applications: A review. Critical Reviews in Solid State and Materials Sciences. 35 (2010) 52-71. https://doi.org/10.1080/10408430903505036.

3. Kulkarni G.S., Reddy K., Zhong Z., Fan X. Graphene nanoelectronic heterodyne sensor for rapid and sensitive vapour detection. Nat Commun. 2014. 4376. P. 1-7; doi: 10.1038/ncomms5376.

4. Karim M.R., Hatakeyama K., Matsui T. et al. Graphene oxide nanosheet with high proton conductivity. J. Am. Chem. Soc. 2013. 135. P. 8097-8100; doi: 10.1021/ja401060q.

5. Xinglin $\mathrm{Yu}$, Xiangdong Chen, Xing Ding. Highsensitivity and low-hysteresis humidity sensor based on hydrothermally reduced graphene oxide/nanodiamond. Sensors and Actuators, B: Chem. 2019. 283. P. 761-768; doi: 10.1016/j.snb.2018.12.057.

6. Prezioso S., Perrozzi F., Giancaterini L., Cantalini C., Treossi E., Palermo V., Nardone M., Santucci S., Ottaviano L. Graphene oxide as practical solution to high sensitivity gas sensing. J. Phys. Chem. C. 2013. 117, No 20. P. 10683-10690; doi: 10.1021/jp3085759.

7. Hwang S., Lim J., Park H.G. et al. Chemical vapor sensing properties of graphene based on geometrical evaluation. Current Appl. Phys. 2012. 12. P. 10171022. DOI: 10.1016/j.cap.2011.12.021.

8. Pandey P.A., Wilson N.R., Covington J.A. Pddoped reduced graphene oxide sensing films for $\mathrm{H}_{2}$ detection. Sensors and Actuators, B: Chem. 2013. 183. P. $478-487$.

http://dx.doi.org/10.1016/j.snb.2013.03.089.

9. Wu Z.-S., Ren W., Gao L., Liu B., Jiang C., Cheng H.-M. Synthesis of high-quality graphene with a pre-determined number of layers. Carbon. 2009. 47. P. 493-499. DOI: 10.1016/j.carbon.2008.10.031.

10. Stankovich S., Dikin D.A., Piner R.D. et al. Synthesis of graphene-based nanosheets via chemical reduction of exfoliated graphite oxide. Carbon. 2007. 45. P. 1558-1565. DOI: 10.1016/j.carbon.2007.02.034.

11. Jung I., Dikin D.A., Piner R.D., Ruoff R.S., Tunable electrical conductivity of individual graphene oxide sheets reduced at "low" temperatures. Nano Lett., 2008. 8, No. 12. P. 42834287. DOI:10.1021/nl8019938.

12. Hummers W.S., Offeman R.E. Preparation of graphitic oxide. J. Am. Chem. Soc. 1958. 80, No 6. P. 1339-1339. DOI: 10.1021/ja01539a017.

13. Slobodian O.M., Lytvyn P.M., Nikolenko A.S. et al. Low-temperature reduction of graphene oxide: electrical conductance and scanning Kelvin probe force microscopy. Nanoscale Res. Lett. 2018. 13, No 1. P. 139; doi: 10.1186/s11671-018-2536-z.

14. Yanga D., Velamakannia A., Bozoklu G. et al. Chemical analysis of graphene oxide films after heat and chemical treatments by X-ray photoelectron and micro-Raman spectroscopy. Carbon. 2009. 47. P. 145-152; doi: 10.1016/j.carbon.2008.09.045.

15. Fu C., Zhao G., Zhang H., Li S. Evaluation and characterization of reduced graphene oxide nanosheets as anode materials for lithium-ion batteries. Int. J. Electrochem. Sci. 2013. 8, N5. P. 6269-6280.

16. Gilje S., Han S., Wang M., Wang K.L., and Kaner R.B. A chemical route to graphene for device applications. Nano Lett. 2007. 7, No 11. P. 339433988. DOI: 10.1021/n10717715.

17. Claramunt S., Varea A., Lopez-Diaz D. et al. The importance of interbands on the interpretation of the Raman spectrum of graphene oxide. J. Phys. Chem. 2015. 119, No 18. P. 10123-10129.

DOI: $10.1021 /$ acs.jpcc.5b01590.

18. Naik G., Krishnaswamy S. Room-temperature humidity sensing using graphene oxide thin films. Graphene. 2016. 5. P. 1-13.

DOI: $10.4236 /$ graphene.2016.51001.

19. Gautam M., Jayatissa A.H. Detection of organic vapors by graphene films functionalized with metallic nanoparticles. J. Appl. Phys. 2012. 112. P. 114326. https://doi.org/10.1063/1.4768724.

20. Gautam M., Jayatissa A.H. Ammonia gas sensing behavior of graphene surface decorated with gold nanoparticles. Solid-State Electron. 2012. 78. P. 159 -165. http://dx.doi.org/10.1016/j.sse.2012.05.059.

\section{Authors and CV}

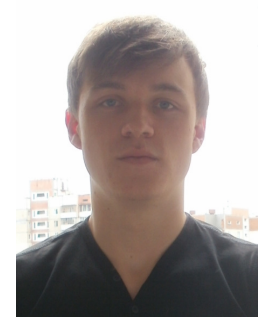

Slobodian O.M., junior research scientist of Department of the Functional Materials and Nanostructures at the V. Lashkaryov Institute of Semiconductor Physics. The area of scientific interests includes investigation of electrical and optical properties of ultrathin carbon films.

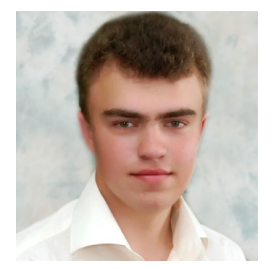

Malyuta S.V. PhD student at the Microelectronics department of National Technical University of Ukraine "Igor Sikorsky Kyiv Polytechnic Institute". The area of scientific interests includes scanning probe microscopy. 


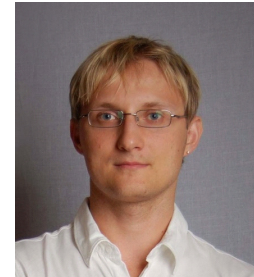

Yurii Milovanov completed his $\mathrm{PhD}$ at Taras Shevchenko National University of Kyiv in Ukraine in 2015. The subject of his research was the investigation of electrical and luminescent properties of porous silicon and titanium oxide composite structures. Currently his research interests focus on the study of nanocomposite structures for chemical gas sensors.

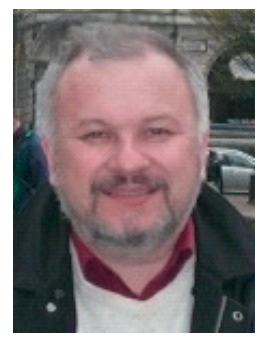

Valery Skryshevsky received his Ph.D. degree in 1984, and his Doctor of Science degree (Habilitation) in 2001 at Taras Shevchenko National University of Kyiv. He is currently professor and Head of Department of Nanophysics of Condensed Matters at Institute of High Technologies, Taras

Shevchenko National University of Kyiv. His main scientific and research interests include micro- and nanotechnology, optoelectronics, solar cells, hydrogen energy, chemical and bio-sensors, development of novel methods of cell imaging using semiconductor nanoparticles.

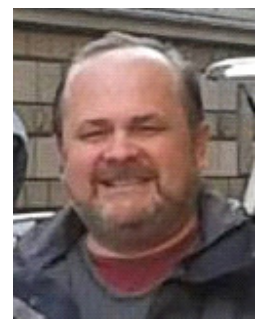

Andrii Vasin received his Ph.D. degree in 2000, and his Doctor of Science degree (Habilitation, solid state physics) in 2016 at V. Lashkaryov Institute of Semiconductor Physics, NASU. He is currently leading research fellow of the department of Functional Materials and Structures of V. Lashkaryov Institute of Semiconductor Physics. His main scientific and research interests include the material science and technology of the functional materials, primarily carbon related nanocomposites and thin films.

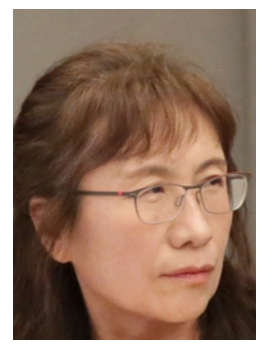

Xiaohui Tang received the Ph.D. degree in Applied Sciences from Université Catholique de Louvain, Belgium in 2001. From 1983 to 1984 , she was an assistant and worked at Kunming University of Technology, China. From 1988 to 1994, she joined at Kunming Institute of Physics, China, where she had been working on II-VI compound semiconductor materials and devices. She then was a free researcher at IMEC, Leuven, Belgium in 1995. Presently, she is working at ICTEAM institute, Université catholique de Louvain, Belgium as a Senior Researcher. She has 128 publications in international journals and conferences, five book chapters and five patents. Her current interest is in fabrication and characterization gas sensors and smart prototypes.

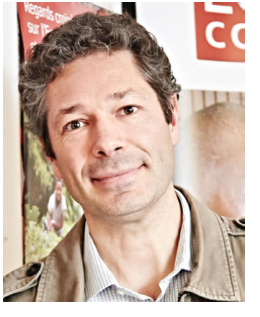

Jean-Pierre Raskin received the M.S. and Ph.D. degrees in applied sciences from Université catholique de Louvain (UCLouvain), Louvain-laNeuve, Belgium, in 1994 and 1997, respectively. He has been a Professor at the Electrical Engineering Department of UCLouvain since 2000. His

research interests are the modeling, wideband characterization and fabrication of advanced SOI MOSFETs as well as micro and nanofabrication of MEMS/NEMS sensors and actuators, including the extraction of intrinsic material properties at nanometer scale. He has been IEEE Fellow since 2014. He was the recipient of the Médaille BLONDEL 2015, the SOI Consortium Award 2016 and the European SEMI Award 2017 in recognition in his vision and pioneering work for RF SOI.

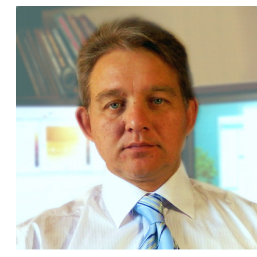

Lytvyn P.M. PhD in Physics and Mathematics, Senior Researcher of the Laboratory of Electron probe methods of structural and elemental analysis of semiconductor materials and systems, V. Lashkaryov Institute of Semiconductor Physics, NAS of Ukraine. The area of scientific interests covers nanophysics of semiconductors and related materials.

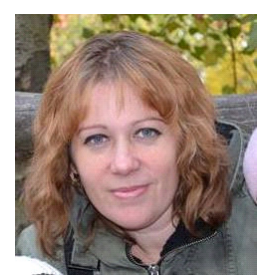

Svezhentsova K.V. PhD in Physics and Mathematics, Senior Researcher of the Department of Physics and Technology of low-dimensional systems, V. Lashkaryov Institute of Semiconductor Physics, NASU. The area of scientific interests includes experimental study of the heterostructures properties applied to detectors of IR and terahertz radiation.

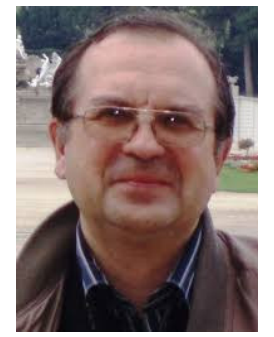

Nazarov A.N. Doctor of Sciences in Physics and Mathematics, Head of Department of the Functional Materials and Nanostructures at the V. Lashkaryov Institute of Semiconductor Physics, NAS of Ukraine, Professor of Department of General Physics and Solid State Physics, National Technical University of Ukraine "Igor Sikorsky KPI".

The area of his scientific interests includes physics, technology and characterization of nanoscaled carbon based materials and devices, SOI structures and devices, radiation effects in carbon and silicon based materials, structures and devices. 